\title{
Prediction on Phase Stabilities of the Zr-H System from the First-Principles
}

\author{
Miao Chen ${ }^{1} \cdot$ Wu Qin ${ }^{2} \cdot$ Yixuan $\mathrm{Hu}^{2} \cdot$ Yiren $\mathrm{Wang}^{2} \cdot$ Yong Jiang ${ }^{2} \cdot$ Xiaosong Zhou $^{1} \cdot$ Shuming Peng ${ }^{1} \cdot$ Yibei Fu $^{1}$
}

Received: 12 April 2020 / Revised: 19 May 2020 / Accepted: 3 June 2020 / Published online: 14 August 2020

(c) The Chinese Society for Metals (CSM) and Springer-Verlag GmbH Germany, part of Springer Nature 2020

\begin{abstract}
Basic fundamentals governing the hydrogenation of $\mathrm{Zr}$ and its alloys have both theoretical and practical importance. In this work, first-principles calculations have been performed to evaluate the relative stabilities of various possible phases in $\mathrm{ZrH}_{x}$ $(x=1-2)$ under different temperatures and pressures. It was predicted that fct- $\gamma$ and $\varepsilon$ phases with various different $\mathrm{H}$-atom configurations can be energetically favorable for $\mathrm{ZrH}_{x}\left(x=1,1.25\right.$ and 1.5), while $\mathrm{ZrH}_{1.75}$ and $\mathrm{ZrH}_{2}$ prefer fct- $\varepsilon$ phase only. Fcc- $\delta$ phase is less favored in energy at any $\mathrm{H}$ concentrations, but can be mechanically stable in some cases. The thermodynamically stable and metastable phase stability diagrams were then constructed for a wide temperature and $\mathrm{H}$ concentration range, to predict the environment-dependent formation of $\mathrm{ZrH}_{x}$ during hydrogenation.
\end{abstract}

Keywords $\mathrm{Zr}-\mathrm{H} \cdot$ Hydrogenation $\cdot$ Hydride $\cdot$ Phase stability $\cdot$ First-principles

\section{Introduction}

The interaction between zirconium $(\mathrm{Zr})$ and hydrogen $(\mathrm{H})$ has attracted increasing research attention over recent decades, from both fundamental and applied perspectives. $\mathrm{Zr}$ and its alloys are widely used as neutron moderators [1] and fuel cladding materials in the nuclear industry, due to their unique combination of low thermal-neutron capture crosssection, good mechanical properties, and high resistance to corrosion [2-4]. They are also found promising applications in storing hydrogen and its isotopes due to their high hydrogen capacities, low equilibrium pressure, and acceptable helium retention rate [5]. However, various stable and metastable phases of zirconium hydrides $\left(\mathrm{ZrH}_{x}\right)$ may precipitate from the $\mathrm{Zr}$ matrix under excess hydrogen exposure, such as in fusion application environment, which can lead to significant embrittlement and thermal-property degradation

Yong Jiang

yjiang@csu.edu.cn

Shuming Peng

pengshuming@caep.cn

1 Institute of Nuclear Physics and Chemistry, China Academy of Engineering Physics, Mianyang 621999, China

2 State Key Laboratory for Powder Metallurgy, School of Materials Science and Engineering, Central South University, Changsha 410083, China
[6]. Basic fundamentals for controlling the hydrogenation of $\mathrm{Zr}$ and its alloys have therefore both theoretical and practical importance.

The $\mathrm{Zr}-\mathrm{H}$ system is known for their complex phase transformations, due to the significant impacts of interstitial $\mathrm{H}$ on the Zr-based crystal lattices. Generally, as the composition $x$ increases from 1 to 2, the face-centered-tetragonal (fct, $c / a>1$ ) $\gamma$, the face-centered-cubic (fcc) $\delta$ and the fct $(c / a<1) \varepsilon$ phases with various different $\mathrm{H}$-atom configurations may form accordingly. A great number of studies on phase transitions exist in the literature, mostly on the $\delta \rightarrow \varepsilon$ transformation of $\mathrm{ZrH}_{x}$. It was found that the crystallographic relation of fcc-to-fct $(\delta \rightarrow \varepsilon)$ transformation can be described well using the phenomenological theory of martensite transformation [7]. Studies on hydrogen diffusion in $\delta$ and $\varepsilon$ suggested that the $\delta \rightarrow \varepsilon$ transformation occurs in the $\mathrm{H}$ concentration range of $1.65 \leq x \leq 1.75$, depending on the temperatures [8-14]. Studies on the $\delta \rightarrow \varepsilon$ lattice distortion using photoelectron spectroscopy or X-ray diffraction suggested that the $\delta \rightarrow \varepsilon$ transformation may occur within $1.63 \leq x \leq 2.0$ [15-18]. As for the fct $\gamma$ phase $(c / a>1)$, it has been generally accepted as a metastable phase of $\mathrm{ZrH}_{x}$ at $x=\sim 1[13,19]$. It can also coexist with hcp $\alpha$-Zr and fcc $\delta-\mathrm{ZrH}_{x}$ within a wide $\mathrm{H}$ concentration range from $x=\sim 0.61$ [20] up to $1.50-1.60$ [1, 18, 21-23]. The $\mathrm{Zr}-\mathrm{H}$ equilibrium phase diagram has been constructed in Ref. [24], but to 
date, it has been still a topic of extensive studies with many debates [11, 25-29].

First-principles calculations have been resorted to explore the electronic structures and energetics of $\mathrm{ZrH}_{x}$. Most of them were focused on fct $\mathrm{ZrH}_{2}$ [27, 29-32], and only a few on phase transitions [33, 34]. A density functional theory (DFT) calculation [29] suggested double local energy minima for fct $\mathrm{ZrH}_{2}$, one existing at $c / a<1$ and the other at $c / a>1$, with a small energy difference of $\sim 0.1 \mathrm{eV}$. The ground state of fct $\mathrm{ZrH}_{2}$ was predicted to be $\gamma$ phase with $c / a>1$, which certainly disagrees with experimental observations [9-11, 35]. Full-potential calculations later confirmed the ground state of fct $\mathrm{ZrH}_{2}$ with $c / a<1$, and based on which, an energetic description was developed for understanding the tetragonal distortion from fcc to fct $\mathrm{ZrH}_{2}$ $[32,36]$. Other a few work focusing on electronic structure properties suggested that $\mathrm{Zr}$ hydrides retain metallic bonding over the whole composition range [14].

In this study, we employed first-principles DFT calculations to revisit the structural stabilities of various possible hydrides in the binary $\mathrm{Zr}-\mathrm{H}$ system at the atomic scale. The atomic structures, mechanical and structural stabilities, and phase transition of $\mathrm{ZrH}_{x}$ within a wide concentration range $(1 \leq x \leq 2)$ were thoroughly investigated and compared. Finally, the phase stability diagrams of the $\mathrm{Zr}-\mathrm{H}$ system subjected to different temperatures and hydrogen pressures were constructed for guiding the hydrogenation practices.

\section{Computational method}

All first-principles DFT calculations were performed using the semi-commercial code-Vienna Ab-initio Simulation Package (VASP) [37, 38] with the plane-wave basis sets and periodic boundary conditions. The Blöchl projector augmented wave method (PAW) within the frozen-core approximation [39] was used to describe the ion-electron interactions. The plane-wave basis sets were generated with valence configurations of $\mathrm{Zr}-4 s^{2} 4 p^{6} 4 d^{2} 5 s^{2}$ and $\mathrm{H}-1 s^{1}$. The exchange-correlation functionals were treated within the generalized gradient approximation (GGA) of Perdew-Burke-Ernzerhof (PBE) [40]. Convergence tests were conducted with respect to the kinetic energy cutoff of the plane-wave basis and the $k$-mesh size for Brillouin-zone integration. A high energy cutoff of $490 \mathrm{eV}$ and a $7 \times 7 \times 7$ Monkhorst-Pack $k$-mesh was found to be sufficient for bulk calculations. For all the bulk calculations, the self-consistence convergence criterion for electron iterations was set to $10^{-5} \mathrm{eV} /$ atom, and the ground-state atomic geometries were optimized by minimizing the Hellman-Feynman force until the total force on each ion was converged to $0.01 \mathrm{eV} / \AA$.

\section{Results and discussion}

\subsection{Bulk calculations of $\mathrm{ZrH}_{x}$}

Inelastic neutron scattering analyses suggested that $\mathrm{H}$ atoms tend to occupy the tetrahedral-interstitial sites in $\mathrm{Zr}$ [41]. To calculate fcc or fct $\mathrm{ZrH}_{x}$, the 4-atom conventional cell of fcc in Fig. 1 is employed as the starting structure, where all the tetrahedral-interstitial sites are purposely lettered as $a$ to $h$. A total number of 4, 5, 6, 7 or $8 \mathrm{H}$ atoms are assigned to these interstitial sites, to construct the $\mathrm{ZrH}_{x}$ supercell with a composition of $x=1,1.25,1.5,1.75$ or 2 , respectively. For instance, the [-adeh-] structure of $\mathrm{ZrH}$ indicates that the four $\mathrm{H}$ atoms are assigned to the interstitial sites of $a, d, e$, and $h$. We first predicted the bulk properties of various $\mathrm{ZrH}_{x}$ $(x=1,1.25,1.5,1.75,2)$, such as lattice constants and bulk moduli, by fitting the calculated total energy versus volume data into the Murnaghan's equation of state. For each composition, all possible configurations of $\mathrm{H}$ atoms have been considered, and further for each $\mathrm{H}$-atom configuration, the $c / a$ value of the supercell varied from 0.80 to 1.20 with an interval of 0.02 , to search for the minimal energy structure through full relaxation calculations. The formation energy of $\mathrm{ZrH}_{x}$ can be calculated as

$\Delta E^{f}=\frac{1}{x+1}\left[\mu\left(\mathrm{ZrH}_{x}\right)-\mu(\mathrm{Zr})-\frac{x}{2} \mu\left(\mathrm{H}_{2}\right)\right]$,

where $\mu\left(\mathrm{ZrH}_{x}\right), \mu(\mathrm{Zr})$ and $\mu\left(\mathrm{H}_{2}\right)$ are the chemical potential of $\mathrm{ZrH}_{x}, \alpha-\mathrm{Zr}$ and $\mathrm{H}_{2}$ molecule, respectively. All the terms are temperature dependent, especially the $\mathrm{H}_{2}$ term. But nevertheless, we are targeted to compare the relative stabilities of different $\mathrm{H}$-atom configurations under the same $\mathrm{H}$ concentration of $x$, and thus all these terms can be calculated at

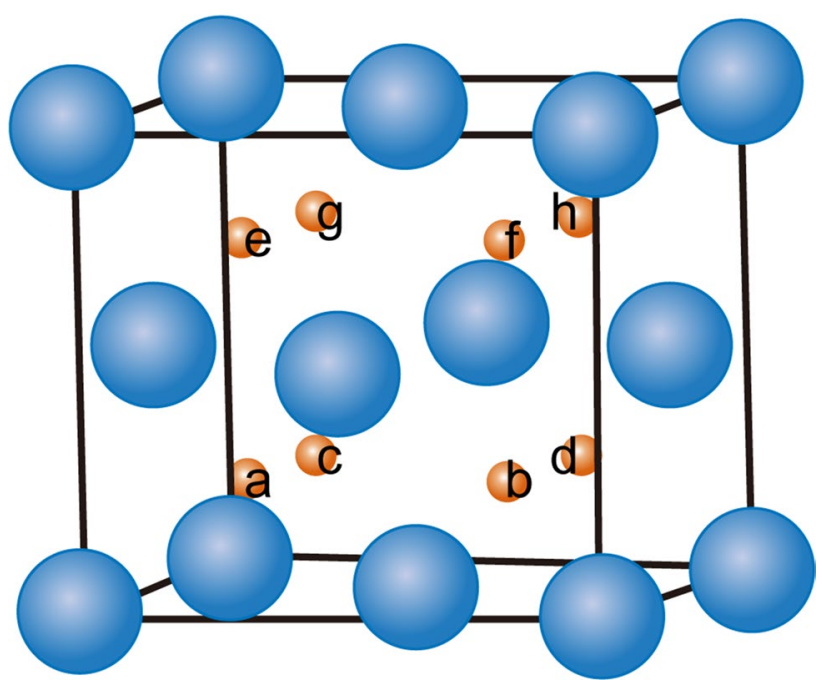

Fig. 1 Potential interstitial sites for $\mathrm{H}$ in bulk $\mathrm{ZrH}_{x}$ 
$0 \mathrm{~K}$, as the first order of approximation. Table 1 summarizes the calculated lattice constants and formation energies for $\mathrm{ZrH}_{x}(x=1,1.25,1.5,1.75,2)$, each with all possible $\mathrm{H}$-atom configurations after full relaxation.

As seen in Table 1, while only one $\mathrm{H}$-atom configuration is possible for $\mathrm{ZrH}_{1.75}$ and $\mathrm{ZrH}_{2}$ due to the supercell symmetry, there are ten, five, and five kinds of $\mathrm{H}$-atom configurations to be considered for $\mathrm{ZrH}, \mathrm{ZrH}_{1.25}$ and $\mathrm{ZrH}_{1.5}$, respectively. We must note here that the $c / a$ values listed in Table 1 are measured only for fct structures, i.e. $\gamma(c / a>1)$ or $\varepsilon(c / a<1)$. A few cases exist that full relaxation may break the initial fct symmetry, leading to a final orthorhombic structure $(a \neq b \neq c)$. However, such orthorhombic structures are not the most energy-favored as predicted in Table 1, nor have been reported in any experiments. Thus, for these cases, we reported the optimized lattice constants only and did not evaluate the $c / a$ ratio in Table 1. Major findings can be summarized as follows. First, all the $\mathrm{H}$-atom configurations were found as energy-favored in $\mathrm{ZrH}_{x}$, with a negative formation energy calculated at ground states. Second, $\mathrm{ZrH}_{1.25}$ can only
Table 1 Predicted lattice constants, formation energies and phase types for $\mathrm{ZrH}_{x}$ with various different $\mathrm{H}$-atom configurations

\begin{tabular}{|c|c|c|c|c|c|c|c|}
\hline $\begin{array}{l}\text { Hydride } \\
\text { composi- } \\
\text { tion }\end{array}$ & $\mathrm{H}$ atom configuration & $\Delta E^{\mathrm{f}}$ (eV/avg atom) & $a(\AA)$ & $b(\AA)$ & $c(\AA)$ & $c / a$ & Predicted phase \\
\hline \multirow[t]{14}{*}{$\mathrm{ZrH}$} & adeh & -0.417 & 4.589 & 4.589 & 5.026 & 1.095 & $\gamma$ \\
\hline & cdef & -0.416 & 4.588 & 4.588 & 5.025 & 1.097 & $\gamma$ \\
\hline & aefh & -0.377 & 4.874 & 4.467 & 4.879 & 1.001 & - \\
\hline & cdfh & -0.376 & 4.880 & 4.880 & 4.463 & 0.915 & $\varepsilon$ \\
\hline & abfg & -0.373 & 4.627 & 4.801 & 4.805 & 1.039 & - \\
\hline & defg & -0.373 & 4.820 & 4.820 & 4.594 & 0.953 & $\varepsilon$ \\
\hline & efgh & -0.337 & 4.616 & 4.616 & 4.999 & 1.083 & $\gamma$ \\
\hline & cdgh & -0.337 & 5.000 & 4.582 & 4.648 & 0.930 & - \\
\hline & bceh & -0.335 & 5.176 & 5.176 & 3.921 & 0.758 & $\varepsilon$ \\
\hline & abce & -0.323 & 5.039 & 5.039 & 4.162 & 0.826 & $\varepsilon$ \\
\hline & Expt. [14] & - & 4.60 & 4.60 & 4.97 & 1.080 & $\gamma$ \\
\hline & Expt. [42] & - & 4.596 & 4.596 & 4.969 & 1.081 & $\gamma$ \\
\hline & GGA. [33] & - & 4.61 & 4.61 & 5.04 & 1.093 & $\gamma$ \\
\hline & GGA. [34] & - & 4.58 & 4.58 & 5.04 & 1.100 & $\gamma$ \\
\hline \multirow[t]{6}{*}{$\mathrm{ZrH}_{1.25}$} & bcdfg & -0.452 & 4.624 & 4.624 & 5.036 & 1.089 & $\gamma$ \\
\hline & bdefg & -0.451 & 4.635 & 4.635 & 5.014 & 1.082 & $\gamma$ \\
\hline & defgh & -0.420 & 4.699 & 4.699 & 4.919 & 1.047 & $\gamma$ \\
\hline & bdefh & -0.420 & 4.644 & 4.913 & 4.760 & 1.025 & - \\
\hline & adefg & -0.413 & 4.739 & 4.739 & 4.861 & 1.026 & $\gamma$ \\
\hline & GGA [33] & - & 4.79 & 4.79 & 5.20 & 1.086 & $\gamma$ \\
\hline \multirow[t]{9}{*}{$\mathrm{ZrH}_{1.5}$} & acdefh & -0.494 & 5.024 & 5.024 & 4.290 & 0.854 & $\varepsilon$ \\
\hline & abcdef & -0.487 & 4.778 & 4.806 & 4.786 & 1.002 & - \\
\hline & bcdfgh & -0.486 & 4.778 & 4.778 & 4.815 & 1.008 & $\gamma$ \\
\hline & abcefh & -0.483 & 4.893 & 4.667 & 4.815 & 0.984 & - \\
\hline & bcefgh & -0.483 & 4.744 & 4.744 & 4.888 & 1.030 & $\gamma$ \\
\hline & Expt. [9] & - & 4.65 & 4.65 & 4.96 & 1.067 & $\gamma$ \\
\hline & GGA [33] & - & 4.62 & 4.62 & 4.83 & 1.046 & $\gamma$ \\
\hline & GGA [33] & - & 5.03 & 5.03 & 4.28 & 0.851 & $\varepsilon$ \\
\hline & GGA [34] & - & 4.61 & 4.61 & 5.14 & 1.115 & $\gamma$ \\
\hline \multirow[t]{3}{*}{$\mathrm{ZrH}_{1.75}$} & abcdefg & -0.525 & 4.936 & 4.936 & 4.545 & 0.921 & $\varepsilon$ \\
\hline & Expt. [43] & - & 4.91 & 4.91 & 4.52 & 0.921 & $\varepsilon$ \\
\hline & GGA [33] & - & 4.97 & 4.97 & 4.47 & 0.899 & $\varepsilon$ \\
\hline \multirow[t]{5}{*}{$\mathrm{ZrH}_{2}$} & abcdefgh & -0.556 & 5.006 & 5.006 & 4.439 & 0.887 & $\varepsilon$ \\
\hline & Expt. [43] & - & 4.980 & 4.980 & 4.430 & 0.890 & $\varepsilon$ \\
\hline & Expt. [11] & - & 4.983 & 4.983 & 4.449 & 0.893 & $\varepsilon$ \\
\hline & GGA [29] & - & 5.000 & 5.000 & 4.450 & 0.890 & $\varepsilon$ \\
\hline & GGA [44] & - & 5.020 & 5.020 & 4.430 & 0.882 & $\varepsilon$ \\
\hline
\end{tabular}


have an fct $\gamma$ phase $(c / a>1)$, while $\mathrm{ZrH}_{1.75}$ and $\mathrm{ZrH}_{2}$ can only have an fct $\varepsilon$ phase. $\mathrm{ZrH}$ and $\mathrm{ZrH}_{1.5}$ both can have fct $\gamma$ and $\varepsilon$ phases. More specifically, fct $\gamma$ or $\varepsilon$ was predicted as the most stable structure for $\mathrm{ZrH}$ or $\mathrm{ZrH}_{1.5}$, respectively, but nevertheless, the energy difference between fct $\gamma$ and $\varepsilon$ was rather small, only $\sim 1.6 \%$ for $\mathrm{ZrH}_{1.5}$ and $\sim 9.8 \%$ for $\mathrm{ZrH}$. Moreover, we must note that fcc- $\delta$ also has negative formation energy for all the $\mathrm{H}$ concentrations in Table 1, but no fcc- $\delta$ phase is predicted as the energy-minimal structure. In other words, fcc $\delta$ phase is not so favorable by energy as the other two fct phases. During relaxation, the initial symmetry can be also broken by Jahn-Teller type distortion, as previously suggested for the case of $\mathrm{ZrH}_{2}$ [30]. In next section, we further performed elastic property calculations on all these hydrides, to evaluate their structural stabilities in view of elastic mechanical performance.

\subsection{Mechanical stability analyses of $\mathrm{ZrH}_{x}$}

Elastic constants determine the elastic response of a crystal to external stress, and are thus often used to assess the mechanical stabilities of a crystal subject to local elastic distortion [45]. For a cubic phase with three independent elastic constants, i.e., $C_{11}, C_{12}$, and $C_{44}$, the following elastic criteria must be met to ensure its mechanical stability [46], i.e.

$C_{11}>0, C_{44}>0, C_{11}-C_{12}>0,\left(C_{11}+2 C_{12}\right)>0$.

For afct phase, there exist six independent elastic constants, i.e., $C_{11}, C_{12}, C_{13}, C_{33}, C_{44}$, and $C_{66}$, and accordingly, two sets of criteria have been proposed as the necessary and sufficient conditions for mechanical stability, namely.

Criteria I [46]:
The energy-favored phases as we predicted in Table 1 are also noted in brackets.

Energy stability indicates the formation preference of a crystal structure, and mechanical stability measures the mechanical resistance of the crystal structure to lattice distortion. By combining the results of Tables 1 and 2, many useful insights can be suggested as follows. First, the lowest energy $\gamma$-phase $\mathrm{ZrH}$ (with an $\mathrm{H}$-atom configuration of adeh or cdef) is not mechanically stable, and instead, the second lowest energy $\varepsilon$-phase $\mathrm{ZrH}$ (with an $\mathrm{H}$-atom configuration of cdfh or defg) can be mechanically stable. This suggests that under certain circumstances, $\varepsilon$ - $\mathrm{ZrH}$ could form as a metastable phase. Second, for $\mathrm{ZrH}_{1.25}$, all the $\mathrm{H}$-atom configurations favor $\gamma$ phase in energy. They are all mechanically stable too, except the only one of bcdfg. For the case of $\mathrm{ZrH}_{1.5}$, both $\varepsilon$ and $\gamma$ can be expected from both energy and mechanical stability perspectives, although $\varepsilon$ is slightly more favored by energy than $\gamma$. For $\mathrm{ZrH}_{1.75}$ and $\mathrm{ZrH}_{2}, \varepsilon$ phase is the only possible stable structure predicted by energy and also by elastic properties. Last, although it is not the most energypreferred, fcc- $\delta$ phase can be mechanically more stable for $\mathrm{ZrH}_{x}(x<1.75)$, according to Table 2. This finding, combing with the above prediction that some fct phases $(\gamma$ or $\varepsilon$ ) are the most energy-favored but mechanically instable (i.e. with a low resistance to distortion), may help us to better understand many diverse experimental observations, such as the coexistence of fct $\gamma$ and fcc- $\delta \mathrm{ZrH}_{x}$ within a wide concentration range from $x=\sim 0.61$ [20] up to $1.50-1.60$ $[1,18,21-23]$, as well as the coexistence of fct- $\varepsilon$ and fcc- $\delta$ within $1.65 \leq x \leq 1.74$ [8-14].

$C_{11}>\left|C_{12}\right|, C_{33}>0, C_{44}>0, C_{66}>0,\left(C_{11}+C_{33}-2 C_{13}\right)>0,2\left(C_{11}+C_{12}\right)+C_{33}+4 C_{13}>0$,

and Criteria II [47]:

$C_{11}>\left|C_{12}\right|, C_{44}>0, C_{66}>0,2 C_{13}^{2}<C_{33}\left(C_{11}+C_{12}\right)$.

All of these elastic constants can be deduced from a series of second-order polynomial relation between total energy and distortion based on first-principles calculations [48]. The results for $\mathrm{ZrH}_{x}$ with all possible structures, i.e. fct- $\gamma$, fct- $\varepsilon$, and fcc- $\delta$, are summarized in Table 2. Please note that all $C_{i j}$ values in Table 2 are predicted as positive, and thus for $\mathrm{ZrH}_{x}$ phases, the mechanical stability needs to meet the conditions of $C_{11}>C_{12}$ and $C_{11}+C_{33}>2 C_{13}$ only (by Criteria I) or $2 C_{13}^{2}<C_{33}\left(C_{11}+C_{12}\right)$ only (by Criteria II). We are thus able to predict many possible mechanically stable phases of $\mathrm{ZrH}_{x}$ that are marked using underlines in Table 2.

\subsection{Thermodynamics calculations of $\mathrm{ZrH}_{x}$}

In the above sections, we have discussed the energy and mechanical stabilities of various $\mathrm{ZrH}_{x}$ phases at their ground states. To explore the thermodynamic stabilities of these hydrides at more practical conditions, we further evaluated the formation energies of $\mathrm{ZrH}_{x}$ under different temperatures and hydrogen pressures. These results enabled us to construct the phase stability diagram of $\mathrm{ZrH}_{x}$.

First, we can rewrite Eq. (1) to be a more general form as

$\Delta E_{\mathrm{ZrH}_{x}}^{\mathrm{f}}(T, P)=\mu_{\mathrm{ZrH}_{x}}(T)-\mu_{\mathrm{Zr}}(T)-\frac{x}{2} \mu_{\mathrm{H}_{2}}(T, P)$,

where the hydrogen chemical potential $\mu_{\mathrm{H} 2}$ can be expressed as a function of the ambient environmental conditions ( $T$ and $\left.p_{\mathrm{H} 2}\right)$ as [49] 
Table 2 Calculated elastic constants $\left(C_{i j}\right)$ for $\mathrm{ZrH}_{x}$ with various possible phase structures

\begin{tabular}{|c|c|c|c|c|c|c|c|c|}
\hline \multirow{2}{*}{$\begin{array}{l}\text { Hydride } \\
\text { composi- } \\
\text { tion }\end{array}$} & \multirow[t]{2}{*}{$\mathrm{H}$-atom configuration } & \multirow[t]{2}{*}{ Phase } & \multicolumn{6}{|c|}{$C_{i j}(\mathrm{GPa})$} \\
\hline & & & $C_{11}$ & $C_{12}$ & $C_{44}$ & $C_{13}$ & $C_{33}$ & $C_{66}$ \\
\hline \multirow[t]{12}{*}{$\mathrm{ZrH}$} & \multirow[t]{3}{*}{$\operatorname{adeh}(\gamma)$} & $\gamma$ & 117.81 & 120.22 & 45.58 & 95.14 & 176.23 & 55.04 \\
\hline & & $\varepsilon$ & 132.41 & 107.72 & 63.82 & 115.24 & 83.15 & 35.24 \\
\hline & & $\underline{\delta}$ & 113.73 & 109.53 & 54.80 & & & \\
\hline & \multirow[t]{2}{*}{$\operatorname{cdef}(\gamma)$} & $\gamma$ & 118.06 & 95.87 & 45.75 & 120.66 & 118.06 & 55.23 \\
\hline & & $\underline{\delta}$ & 113.71 & 113.51 & 54.85 & & & \\
\hline & \multirow[t]{2}{*}{$\operatorname{cdfh}(\varepsilon)$} & $\underline{\varepsilon}$ & 143.94 & 105.30 & 69.91 & 106.16 & 106.16 & 55.25 \\
\hline & & $\underline{\delta}$ & 114.60 & 106.37 & 57.07 & & & \\
\hline & \multirow[t]{3}{*}{ bceh $(\varepsilon)$} & $\underline{\varepsilon}$ & 184.29 & 100.00 & 63.07 & 96.37 & 103.40 & 29.92 \\
\hline & & $\gamma$ & 99.72 & 120.35 & 67.51 & 111.83 & 144.27 & 81.64 \\
\hline & & $\delta$ & 113.32 & 116.19 & 78.56 & & & \\
\hline & \multirow[t]{2}{*}{$\operatorname{defg}(\varepsilon)$} & $\underline{\varepsilon}$ & 136.62 & 101.07 & 70.05 & 109.56 & 109.60 & 55.07 \\
\hline & & $\underline{\delta}$ & 122.45 & 103.79 & 67.52 & & & \\
\hline \multirow[t]{11}{*}{$\mathrm{ZrH}_{1.25}$} & \multirow[t]{3}{*}{$\operatorname{bcdfg}(\gamma)$} & $\gamma$ & 116.90 & 122.68 & 55.74 & 109.89 & 165.97 & 55.74 \\
\hline & & $\varepsilon$ & 133.03 & 109.88 & 62.76 & 120.12 & 100.20 & 48.20 \\
\hline & & $\delta$ & 114.48 & 114.96 & 52.93 & & & \\
\hline & \multirow[t]{2}{*}{ bdefg $(\gamma)$} & $z^{*}$ & 166.52 & 108.89 & 53.70 & 108.89 & 117.05 & 55.65 \\
\hline & & $\underline{\delta}$ & 127.48 & 117.88 & 57.92 & & & \\
\hline & \multirow[t]{3}{*}{$\operatorname{defgh}(\gamma)$} & $z$ & 130.32 & 117.98 & 76.22 & 104.60 & 104.60 & 80.56 \\
\hline & & $\underline{\varepsilon}^{*}$ & 164.63 & 100.89 & 78.98 & 111.51 & 92.69 & 46.78 \\
\hline & & $\underline{\delta}$ & 133.07 & 102.82 & 79.07 & & & \\
\hline & \multirow[t]{3}{*}{$\operatorname{adefg}(\gamma)$} & $\gamma$ & 119.01 & 120.80 & 66.64 & 114.86 & 136.41 & 73.00 \\
\hline & & $\underline{\varepsilon}^{*}$ & 156.77 & 106.17 & 71.30 & 116.95 & 92.20 & 92.20 \\
\hline & & $\underline{\delta}$ & 124.55 & 117.07 & 69.20 & & & \\
\hline \multirow[t]{7}{*}{$\mathrm{ZrH}_{1.5}$} & \multirow[t]{3}{*}{ acdefh $(\varepsilon)$} & $\underline{\varepsilon}$ & 162.59 & 131.70 & 55.47 & 104.24 & 114.96 & 38.66 \\
\hline & & $\gamma$ & 112.32 & 132.00 & 40.43 & 114.36 & 166.18 & 52.07 \\
\hline & & $\delta$ & 106.80 & 130.43 & 34.99 & & & \\
\hline & \multirow[t]{2}{*}{ bcefgh $(\gamma)$} & z & 127.01 & 123.83 & 58.42 & 118.89 & 154.73 & 60.66 \\
\hline & & $\underline{\delta}$ & 119.11 & 117.76 & 55.98 & & & \\
\hline & \multirow[t]{2}{*}{ bcdfgh $(\gamma)$} & $\not$ & 137.47 & 117.48 & 68.44 & 116.23 & 152.49 & 72.71 \\
\hline & & $\delta$ & 117.57 & 121.60 & 55.76 & & & \\
\hline \multirow[t]{3}{*}{$\mathrm{ZrH}_{1.75}$} & \multirow[t]{3}{*}{ abcdefg $(\varepsilon)$} & $\underline{\varepsilon}$ & 149.50 & 128.09 & 46.20 & 125.02 & 125.02 & 40.05 \\
\hline & & $\gamma$ & 125.13 & 132.54 & 45.09 & 125.49 & 154.20 & 47.48 \\
\hline & & $\delta$ & 122.95 & 131.63 & 32.70 & & & \\
\hline \multirow[t]{6}{*}{$\mathrm{ZrH}_{2}$} & \multirow[t]{3}{*}{ abcdefgh $(\varepsilon)$} & $\underline{\varepsilon}$ & 163.88 & 146.06 & 34.85 & 112.60 & 142.05 & 52.41 \\
\hline & & $\gamma$ & 132.00 & 141.73 & 36.92 & 119.00 & 185.79 & 34.97 \\
\hline & & $\delta$ & 113.85 & 141.78 & 12.85 & & & \\
\hline & \multirow[t]{3}{*}{ GGA [45] $(\varepsilon)$} & $\underline{\varepsilon}$ & 165.6 & 140.9 & 30.5 & 106.8 & 145.5 & 60.6 \\
\hline & & $\gamma$ & 125.7 & 145.5 & 30.9 & 115.0 & 190.6 & 42.0 \\
\hline & & $\delta$ & 82.6 & 159.7 & -19.5 & & & \\
\hline
\end{tabular}

*Predicted as mechanically stable by Criteria I but instable by Criteria II

$\mu_{\mathrm{H}_{2}}(T, P)=\mu_{\mathrm{H}_{2}}^{0}(T)+k_{\mathrm{B}} T \ln \frac{P_{\mathrm{H}_{2}}}{P_{\mathrm{H}_{2}}^{0}},=\mu_{\mathrm{H}_{2}}^{0}(0 \mathrm{~K})+\Delta H_{\mathrm{H}_{2}}(T)-T \Delta S_{\mathrm{H}_{2}}(T)+k_{\mathrm{B}} T \ln P_{\mathrm{H}_{2}}$,

where the superscript " 0 " stands for the stand state. $p_{\mathrm{H}_{2}}^{0}$ is taken to be 1 atm. $k_{\mathrm{B}}$ is the Boltzmann constant,
$1.381 \times 10^{-23} \mathrm{~J} / \mathrm{K}$. Eventually, the formation energy, $\Delta E^{\mathrm{f}}$, 
can be expressed as the function of temperature $(T)$ and hydrogen partial pressure $\left(p_{\mathrm{H} 2}\right)$, as
Figures 2 and 3 compare the calculated formation energies of various stable and metastable $\mathrm{ZrH}_{x}$ phases, respec-

$\Delta E_{\mathrm{ZrH}_{x}}^{\mathrm{f}}(T, P)=\mu_{\mathrm{ZrH}_{x}}(T)-\mu_{\mathrm{Zr}}(T)-\frac{x}{2}\left(\mu_{\mathrm{H} 2}^{0}(0 \mathrm{~K})+\Delta H_{\mathrm{H}_{2}}(T)-T \Delta S_{\mathrm{H}_{2}}(T)+k_{\mathrm{B}} T \ln P_{\mathrm{H}_{2}}\right)$

Please note that the temperature dependences of the chemical potentials of pure solids, i.e. $\mu_{\mathrm{ZrH}_{x}}$ and $\mu_{\mathrm{Zr}}$, would largely cancel out with each other. The major contribution to the temperature dependence of $\Delta E^{\mathrm{f}}$ thus arises from the enthalpic and entropic terms of gaseous molecule $\mathrm{H}_{2}$, i.e. $\Delta H_{\mathrm{H}_{2}}$ and $\Delta S_{\mathrm{H}_{2}}(T)$, which can be referred to experimental values as tabulated in JANAF tables [50]. Therefore, when computing the formation energy using Eq. (6), we were able to approximate $\mu_{\mathrm{ZrH}_{x}}$ and $\mu_{\mathrm{Zr}}$ with DFT results calculated at $0 \mathrm{~K}$.

According to Eqs. (4-6), the formation energy of a $\mathrm{ZrH}_{x}$ phase can no longer be a constant value, but depend on the ambient conditions, i.e. $T$ and $p_{\mathrm{H} 2}$. The upper limit of $p_{\mathrm{H} 2}$ can be determined by requiring no net loss of hydrogen from the bulk under the thermodynamic equilibrium conditions, $\mu_{\mathrm{H}_{2}} \leq \mu_{\mathrm{H}_{2}}^{0}$, i.e. $p_{\mathrm{H}_{2}} \leq 1 \mathrm{~atm}$.
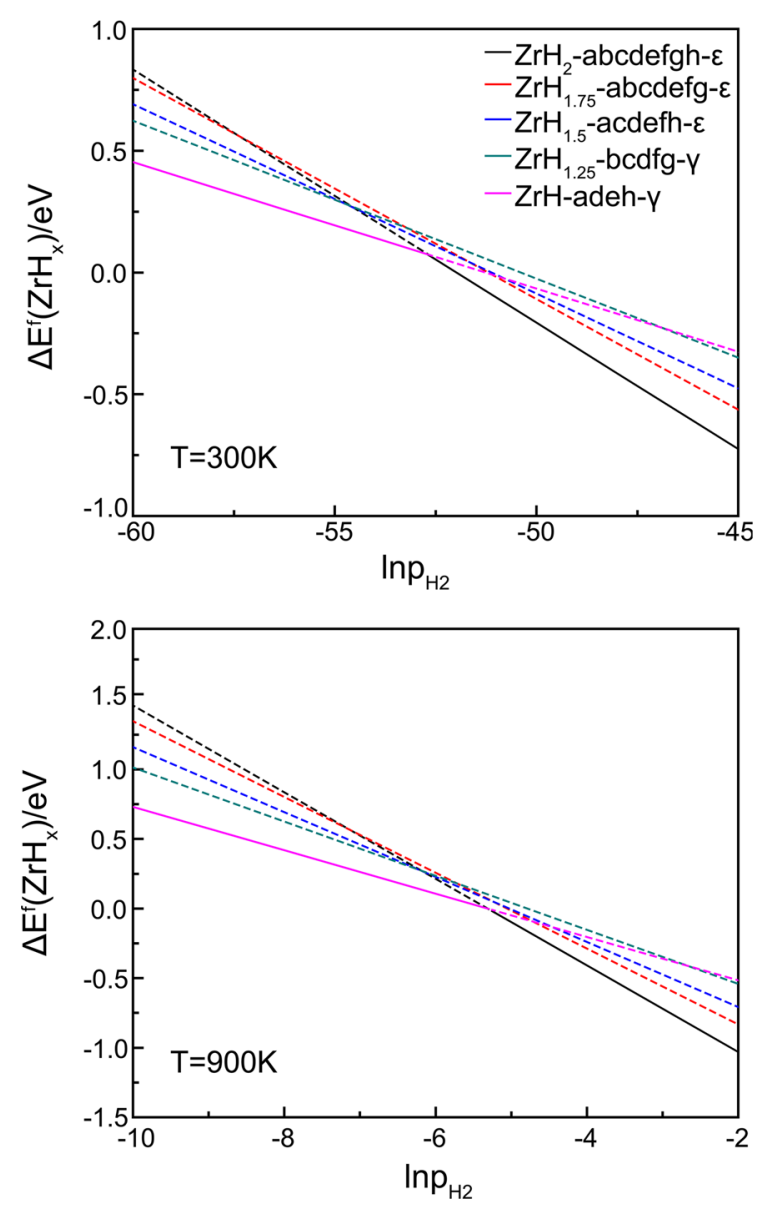

tively, as a function of temperature and hydrogen partial pressure using Eq. (6). Specifically, for the calculations in Fig. 2, we adopted the lowest energy structures of $\mathrm{ZrH}_{x}$ $(x=1,1.25,1.5,1.75$, and 2), each with the lowest energy configuration of $\mathrm{H}$ atoms as predicted in Table 1, i.e. $\mathrm{ZrH}$ adeh- $\gamma, \mathrm{ZrH}_{1.25}$-bcdfg- $\gamma, \mathrm{ZrH}_{1.5}$-acdefh- $\varepsilon, \mathrm{ZrH}_{1.75}$-abcdef $g-\varepsilon$, or $\mathrm{ZrH}_{2}$-abcdefgh- $\varepsilon$, respectively. In Fig. 3, the calculations adopted the metastable $\mathrm{ZrH}_{x}$ phases, each with the lowest energy configuration of $\mathrm{H}$ atoms as predicted in Tables 1 and 2, including $\mathrm{ZrH}$-cdfh- $\varepsilon, \mathrm{ZrH}_{1.25}$-defgh- $\varepsilon$, and $\mathrm{ZrH}_{1.5}$-bcdfgh- $\gamma$. Please notice that $\mathrm{ZrH}_{1.75}$ and $\mathrm{ZrH}_{2}$ have no metastable phases to form, and hence the stable phases of $\mathrm{ZrH}_{1.75}$-abcdef $g$ - $\varepsilon$ and $\mathrm{ZrH}_{2}$-abcdefgh- $\varepsilon$ were adopted. It is clearly seen from Figs. 2 and 3 that the formation energies of these phases $\mathrm{ZrH}_{x}$ always increase with the temperature but decrease with the hydrogen partial pressure. The intersection
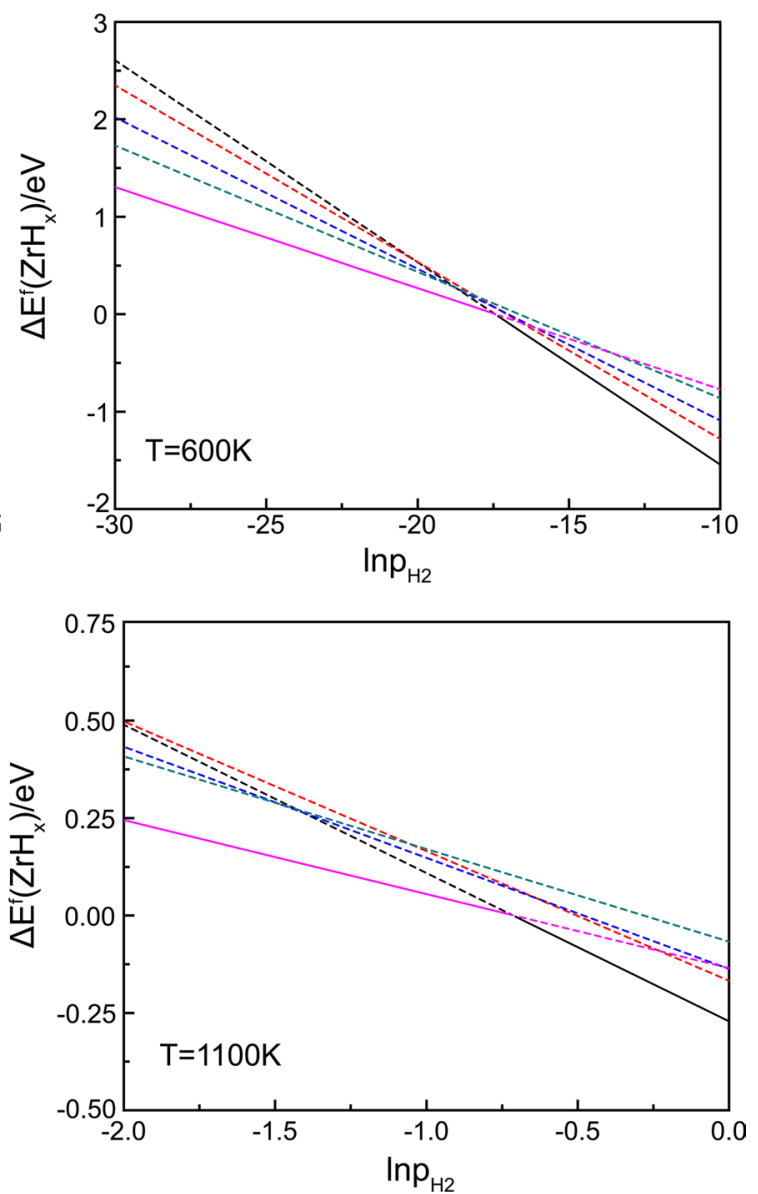

Fig. 2 Predicted formation energies of various stable $\mathrm{ZrH}_{x}$ phases as a function of temperature and hydrogen partial pressure 

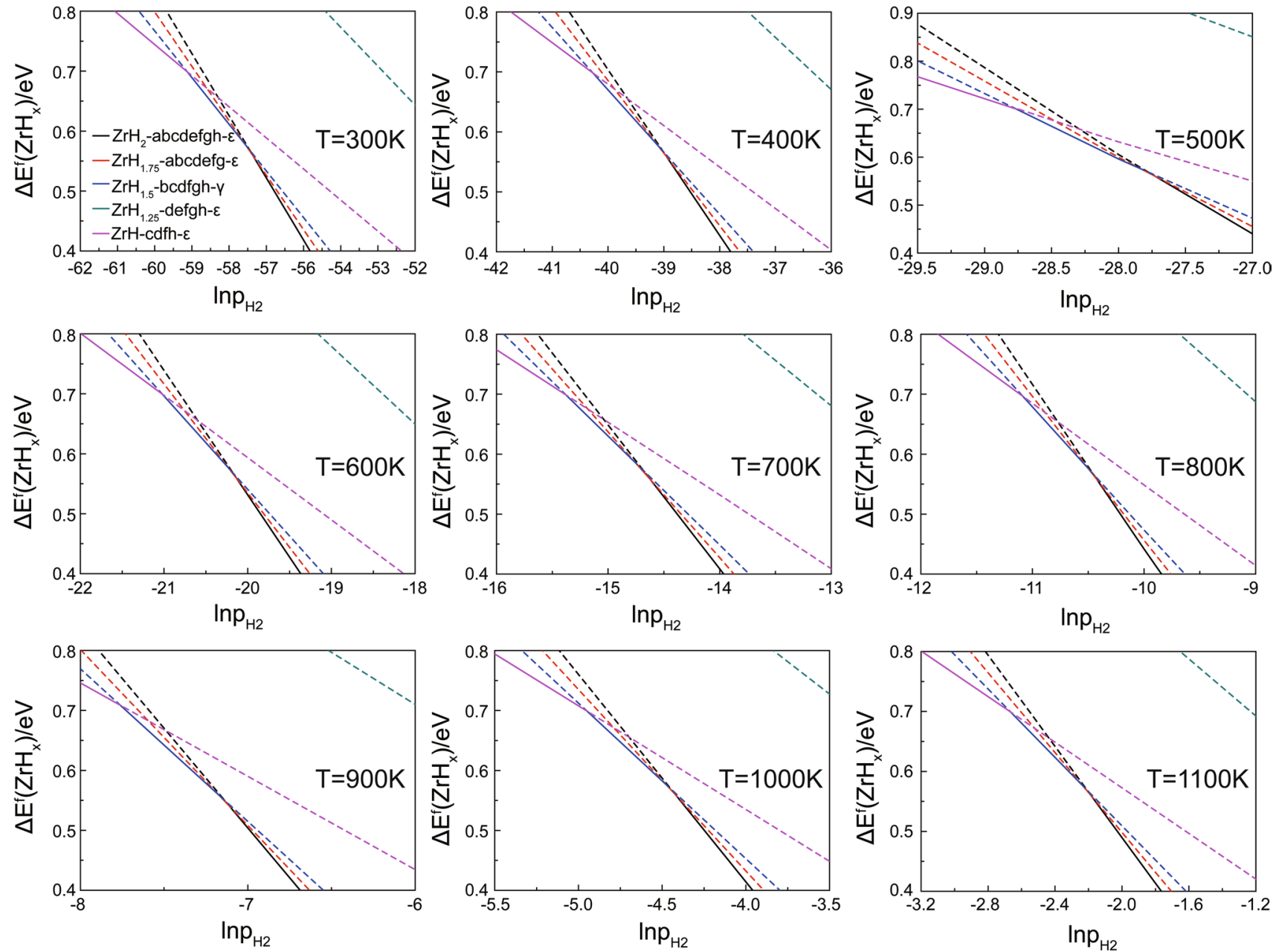

Fig. 3 Predicted formation energies of various metastable $\mathrm{ZrH}_{x}$ phases as a function of temperature and hydrogen partial pressure
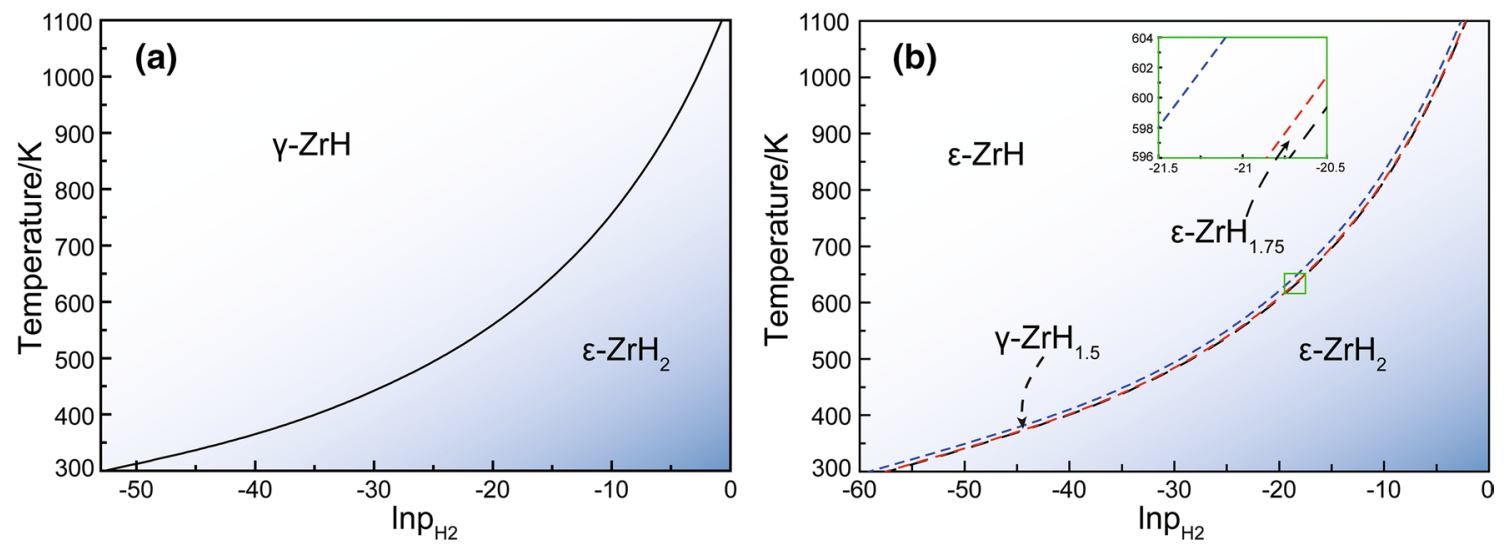

Fig. 4 Calculated stable a and metastable b phase stability diagrams of $\mathrm{ZrH}_{x}$ predicting the relative stabilities of various possible $\mathrm{ZrH}$ phases under a given temperature and hydrogen partial pressure

of any two data lines predicts a phase stability transition which also depends on the temperature and hydrogen partial pressure. Tracking the lowest energy intersections in Figs. 2 and 3 under a given temperature allowed us to construct the phase stability diagrams in Fig. 4, where Fig. 4a is the stable 
phase stability diagram and Fig. 4a the metastable phase stability diagram of $\mathrm{ZrH}_{x}$.

It is revealed in Fig. 2 that, over the whole range of temperature and pressure being considered, $\gamma-\mathrm{ZrH}$ and $\varepsilon-\mathrm{ZrH}_{2}$ phases take the lead having the lowest formation energies, while all other $\mathrm{ZrH}_{x}$ phases have relatively higher formation energies. Thus the only possible phase transition exists between $\gamma$ - $\mathrm{ZrH}$ and $\varepsilon-\mathrm{ZrH}_{2}$, which partitions the stable phase stability diagram into two regions as seen in Fig. 4a. $\gamma$ - $\mathrm{ZrH}$ appears to dominate in higher temperatures and/or lower hydrogen partial pressures, while $\varepsilon-\mathrm{ZrH}_{2}$ appears to dominate in lower temperatures and/or higher hydrogen partial pressures. The phase transition temperature sensitively depends on hydrogen partial pressure. For instance, at $T=300 \mathrm{~K}, \varepsilon-\mathrm{ZrH}_{2}$ becomes more stable than $\gamma-\mathrm{ZrH}$ when $\ln p_{\mathrm{H} 2} \geq 53$. When increasing $p_{\mathrm{H} 2}$, this transition temperature moves to higher (thus more practical) pressures. At $T=1100 \mathrm{~K}$, the transition starts to occur at $\ln p_{\mathrm{H} 2}=\sim-0.75$.

For metastable phases in Fig. 3, more phase transitions were revealed. Consequently, there exist four phase regions in the metastable phase stability diagram in Fig. 4b. Metastable $\varepsilon$ - $\mathrm{ZrH}$ appears to dominate in the high temperature and/or low partial pressure region of the diagram, while two extremely narrow regions of $\gamma-\mathrm{ZrH}_{1.5}$ and $\varepsilon-\mathrm{ZrH}_{1.75}$ appear on the side of $\gamma-\mathrm{ZrH}$ in Fig. 4a. The region for the stable $\varepsilon$ $\mathrm{ZrH}_{2}$ phase expands slightly in the metastable phase stability diagram. At $T=600 \mathrm{~K}$, the $\varepsilon-\mathrm{ZrH}$-to- $\gamma-\mathrm{ZrH}_{1.5}$ transition occurs at $\ln p_{\mathrm{H} 2}=\sim-21.1$, the $\gamma-\mathrm{ZrH}_{1.5}$-to- $\varepsilon-\mathrm{ZrH}_{1.75}$ transition at $\ln p_{\mathrm{H} 2}=\sim-20.3$, and $\varepsilon-\mathrm{ZrH}_{1.75}$-to- $\varepsilon-\mathrm{ZrH}_{2}$ transition at $\ln p_{\mathrm{H} 2}=\sim-20.1$.

In practices, the co-presence of various stable and metastable phases of $\mathrm{ZrH}_{x}$ has been often reported in experiments, and the characterization results usually involve many disputes. This can be strongly related to the facts that accurate measurement and characterization of $\mathrm{H}$ atoms in $\mathrm{ZrH}_{x}$ still face great challenges to date, and that these stable and metastable $\mathrm{ZrH}_{x}$ phases generally have very comparable formation energies (Table 1). Also importantly, $\mathrm{H}$ atoms can diffuse fairly fast among these phases. Thus, the stable and metastable phase stability diagrams in Fig. 4 shall be combined in uses, for predicting the relative stabilities of $\mathrm{ZrH}_{x}$ phases under a given temperature and hydrogen partial pressure. It is suggested in Fig. 4 that the stable $\varepsilon-\mathrm{ZrH}_{2}$ always dominates at lower temperatures and higher partial pressures, the stable $\gamma$ - $\mathrm{ZrH}$ and metastable $\varepsilon$ - $\mathrm{ZrH}$ can co-present at higher temperatures and lower partial pressures, while in the intermediate range, various stable and metastable $\mathrm{ZrH}_{x}$ phases could be expected, including stable $\gamma-\mathrm{ZrH}$ and $\varepsilon$ $\mathrm{ZrH}_{2}$, and metastable $\varepsilon-\mathrm{ZrH}, \gamma-\mathrm{ZrH}_{1.5}$, and $\varepsilon-\mathrm{ZrH}_{1.75}$ as well.

\section{Conclusions}

First-principles modeling and calculations have been performed to predict the relative stabilities of various possible stable and metastable phases in $\mathrm{ZrH}_{x}(x=1,1.25,1.5,1.75$ and 2) at different temperatures and pressures, and some conclusive remarks have been obtained.

1. H configuration has profound effect on the structural and mechanical stability of $\mathrm{ZrH}_{x}$ phases. Various fct $\gamma$ and $\varepsilon$ phases with different $\mathrm{H}$ atom configurations can be energetically favored for $\mathrm{ZrH}_{x}(x=1,1.25$ and 1.5), while $\mathrm{ZrH}_{1.75}$ and $\mathrm{ZrH}_{2}$ only prefer the fct- $\varepsilon$ phase.

2. Some of the most energy-favored phases, such as $\gamma$ $\mathrm{ZrH}$ (-adeh and -cdef) and $\gamma-\mathrm{ZrH}_{1.25}(-\mathrm{bcdfg})$, are not mechanically stable. Fcc- $\delta$ phase is less preferred in energy than other fct phases at any $\mathrm{H}$ concentrations, but can be mechanically more stable in some cases.

3. The formation energies of $\mathrm{ZrH}_{x}$ phases always increase with temperatures, but decrease with hydrogen partial pressures. For the energy stable phases, the only phase transition can be expected between $\gamma-\mathrm{ZrH}$ and $\varepsilon-\mathrm{ZrH}_{2}$ phases, partitioning the stable phase stability diagram into two regions. $\gamma$ - $\mathrm{ZrH}$ turns to dominate at higher temperatures and/or lower hydrogen partial pressures.

4. The metastable phase stability diagram consists of four phase regions of $\varepsilon-\mathrm{ZrH}, \gamma-\mathrm{ZrH}_{1.5}, \varepsilon-\mathrm{ZrH}_{1.75}$ and $\varepsilon-\mathrm{ZrH}_{2}$. The two regions of $\gamma-\mathrm{ZrH}_{1.5}$ and $\varepsilon-\mathrm{ZrH}_{1.75}$ are extremely narrow. $\varepsilon$ - $\mathrm{ZrH}$ turns to dominate at higher temperatures and/or lower hydrogen partial pressures, and $\varepsilon-\mathrm{ZrH}_{2}$ dominates at lower temperatures and/or higher hydrogen partial pressures. In practices, the stable and metastable phase stability diagrams shall be combined in uses, to predict the environment-dependent structures of $\mathrm{ZrH}_{x}$ phases during hydrogenation.

Acknowledgements This work was financially supported by the National MCF Energy R\&D Program of China (Project No. 2018YFE0306100) and the National Natural Science Foundation of China (No. 51971249).

\section{References}

[1] P.W. Bickel, T.G. Berlincourt, Phys. Rev. B 2, 4807 (1970)

[2] K.B. Colas, A.T. Motta, J.D. Almer, M.R. Daymond, M. Kerr, A.D. Banchik, P. Vizcaino, J.R. Santisteban, Acta Mater. 58, 6575 (2010)

[3] A. Singh, P. Kuppusami, R. Thirumurugesan, R. Ramaseshan, M. Kamruddin, S. Dash, V. Ganesan, E. Mohandas, Appl. Surf. Sci. 257, 9909 (2011)

[4] H.O. Pierson, ed. 4 - carbides of group IV: Titanium, zirconium, and hafnium carbides. in Handbook of Refractory Carbides and 
Nitrides (William Andrew Publishing, Westwood, NJ, 1996), pp. $55-80$

[5] G.J. Cheng, G. Huang, M. Chen, X.S. Zhou, J.H. Liu, S.M. Peng, W. Ding, H.F. Wang, L.Q. Shi, J. Nucl. Mater. 499, 490 (2018)

[6] C.E. Ells, J. Nucl. Mater. 28, 129 (1968)

[7] M.P. Cassidy, C.M. Wayman, Metall. Trans. A 11, 57 (1980)

[8] R.C. Bowman, B.D. Craft, J. Phys. C: Solid State Phys. 17, L477 (1984)

[9] R.C. Bowman, E.L. Venturini, B.D. Craft, A. Attalla, D.B. Sullenger, Phys. Rev. B 27, 1474 (1983)

[10] K. Niedźwiedź, B. Nowak, O.J. Żogał, J. Alloys Compd. 194, 47 (1993)

[11] R.C. Bowman Jr., B.D. Craft, J.S. Cantrell, E.L. Venturini, Phys. Rev. B 31, 5604 (1985)

[12] O.J. Żogal, A.H. Vuorimäki, E.E. Ylinen, K. Niedźwiedź, Z. Phys. B: Condens. Matter 96, 293 (1995)

[13] E. Zuzek, J.P. Abriata, A. San-Martin, F.D. Manchester, Bull. Alloy Phase Diagr 11, 385 (1990)

[14] A. Aladjem, Solid State Phenom. 49-50, 281 (1996)

[15] J.S. Cantrell, R.C. Bowman Jr., D.B. Sullenger, J. Phys. Chem. 88, 918 (1984)

[16] J.H. Weaver, D.J. Peterman, D.T. Peterson, A. Franciosi, Phys. Rev. B 23, 1692 (1981)

[17] B.W. Veal, D.J. Lam, D.G. Westlake, Phys. Rev. B 19, 2856 (1979)

[18] K.G. Barraclough, C.J. Beevers, J. Nucl. Mater. 34, 125 (1970)

[19] D.O. Northwood, U. Kosasih, Int. Mater. Rev. 28, 92 (1983)

[20] A.M. Solodinin, E.B. Boyko, R.A. Andriyevskiy, Izv. Akad. Nauk SSSR, Met. 1, 198 (1978) in Russian; TR: Russ. Metall. 1, 178 (1978)

[21] B. Siegel, G.G. Libowitz, Metal Hydrides, Chap. 12, 545 (1968)

[22] S. Mishra, K.S. Sivaramakrihnan, M.K. Asundi, J. Nucl. Mater. 45, 235 (1972)

[23] K.G. Barraclough, C.J. Beevers, J. Less Common Met. 35, 177 (1974)

[24] R.W. Cahn, Adv. Mater. 3, 628 (1991)

[25] F. Ducastelle, R. Caudron, P. Costa, J. Phys. 31, 57 (1970)

[26] M. Gupta, J.P. Burger, Phys. Rev. B 24, 7099 (1981)
[27] A.C. Switendick, J. Less Common Met. 101, 191 (1984)

[28] D.A. Papaconstantopoulos, A.C. Switendick, J. Less Common Met. 103, 317 (1984)

[29] G.J. Ackland, Phys. Rev. Lett. 80, 2233 (1998)

[30] M. Gupta, Phys. Rev. Lett. 81, 3300 (1998)

[31] M. Gupta, Phys. Rev. B 25, 1027 (1982)

[32] R. Quijano, R. de Coss, D.J. Singh, Phys. Rev. B 80, 184103 (2009)

[33] F. Wang, H.R. Gong, Int. J. Hydrog. Energy 37, 9688 (2012)

[34] C. Domain, R. Besson, A. Legris, Acta Mater. 50, 3513 (2002)

[35] H.L. Yakel, Acta Crystall. 11, 46 (1958)

[36] W. Wolf, P. Herzig, J. Phys.: Condens. Matter 12, 4535 (2000)

[37] J. Furthmuller, J. Hafner, G. Kresse, Phys. Rev. B 50, 15606 (1994)

[38] W. Dong, G. Kresse, J. Furthmuller, J. Hafner, Phys. Rev. B 54, 2157 (1996)

[39] G. Kresse, D. Joubert, Phys. Rev. B 59, 1758 (1999)

[40] J. Leese, A.E. Lord, J. Appl. Phys. 39, 3986 (1968)

[41] R. Khodabakhsh, D.K. Ross, J. Phys. F: Met. Phys. 12, 15 (1982)

[42] G.C. Weatherly, Acta Metall. 29, 501 (1981)

[43] J.S. Cantrell, R.C.J. Bowman, D.B. Sullenger, Chem. Inform. 15, 918 (1984)

[44] R. Quijano, R. Decoss, D.J. Singh, Phys. Rev. B 80, 2665 (2009)

[45] P. Zhang, B.T. Wang, C.H. He, P. Zhang, Comput. Mater. Sci. 50, $3297(2011)$

[46] J. F. Nye, Oxford University Press (1958)

[47] F. Mouhat, F.X. Coudert, Phys. Rev. B 90, 224104 (2014)

[48] P.J. Dobson, Phys. Bull. 36, 506 (1985)

[49] Y. Jiang, J.B. Adams, M. van Schilfgaarde, J. Chem. Phys. 123, 64701 (2005)

[50] F.D. Rossini, JANAF thermochemical tables: Stull, D. R. and Prophet, M. U.S. Government Printing Office: Washington. Second edition, 1971. J. Chem. Thermodyn. 4, 509 (1972) 